\title{
A new anti-emission material for manufacturing of cathode-mesh electrodes
}

\author{
I. P. Melnikova ${ }^{\dagger}$ A. V. Lyasnikova, S. V. Maltseva \\ †melnikova_ip@mail.ru \\ Yuri Gagarin State Technical University of Saratov, 77 Politechnicheskaya st., 410054, Saratov, Russia
}

\begin{abstract}
The paper examines the properties of anti-emission materials, in which the mechanism of emission suppression is associated with the decomposition of a $\mathrm{BaO}$ layer. Oxide components diffuse into the surface layer, while barium, as a material with low work function, quickly evaporates from the surface of electrodes composed of anti-emission materials. Electrodes of the cathode-mesh assemblies are made of materials with recrystallization temperature of about $900^{\circ} \mathrm{C}$, which is close to the working grid temperature. The newly developed vanadium based alloy VZrCNb10-1 is characterized by a higher recrystallization temperature of $1250^{\circ} \mathrm{C}$. It is experimentally demonstrated that $\mathrm{VZrCNb} 10-1$ is capable of dissolving more oxygen without changes in the phase composition of the surface and significant changes in the mechanical properties. This alloy has a better ability to maintain the strength and technological characteristics during annealing in the vacuum of $6.65 \cdot 10^{-3} \mathrm{~Pa}$ as compared to hafnium and $\mathrm{ZrNb}-25$ alloy. $\mathrm{VZrCNb} 10-1$ is a high-strength heterophase material with carbide hardening and high strength about $60 \mathrm{~kg} / \mathrm{mm}^{2}$ and has good ductility of about $15 \%$. VZrCNb10-1 allows for manufacturing components with small radii of curvature using deep-drawing methods maintaining fine-grained structure at the bend points with a high yield percentage. The studies show that at temperatures of the working mesh of 800,1000 , and $1100^{\circ} \mathrm{C}$, the tensile strength of the $\mathrm{VZrCNb} 10-1$ alloy is 2.5 - 4 times higher than that of the ZrNb-25 alloy or hafnium. VZrCNb10-1 provides an at least twice enhanced durability of electron guns as, compared to the nodes with hafnium grids.
\end{abstract}

Keywords: anti-emission material, phase composition, strength, shape stability.

\section{Новый антиэмиссионный материал для изготовления электродов катодно-сеточных узлов}

\author{
Мельникова И.П. ${ }^{\dagger}$ Лясникова А. В., Мальцева С. В. \\ Саратовский государственный технический университет имени Гагарина Ю. А., \\ ул. Политехническая 77, 410054, Саратов, Россия
}

\begin{abstract}
Исследованы свойства антиэмиссионных материалов, механизм подавления эмиссии которых связан с разложением слоя $\mathrm{BaO}$. Оксидные компоненты диффундируют в поверхностный слой, при этом барий, материал с низкой работой выхода, быстро испаряется с поверхности электрода из антиэмиссионного материала. В настоящее время электроды катодно-сеточных узлов изготавливают из материалов с температурой рекристаллизации, близкой к температуре работающей сетки $\left(900^{\circ} \mathrm{C}\right)$, в основном, из Hf, сплава ЦН-25 без покрытия, Мо с покрытием из $\mathrm{Zr}$, Ті и пр. Вновь разработанный сплав на основе ванадия ВЦУН10-1 отличается высокой температурой рекристаллизации, равной $1250^{\circ} \mathrm{C}$. Экспериментально показано, что сплав ВЦУН10-1 способен растворять большее количество кислорода без изменения фазового состава поверхности и значительного изменения механических свойств. Сплав обладает лучшей способностью сохранять прочностные и технологические характеристики при отжиге в вакууме $6,65 \cdot 10^{-3}$ Па, чем гафний и сплав ЦН-25. ВЦУН10-1 относится к высокопрочным, гетерофазным материалам с карбидным упрочнением и обладает высокой прочностью, примерно равной 60 кг/мм², при хорошей пластичности около 15\%. Сплав на основе ванадия позволяет изготавливать детали с малыми радиусами кривизны методами глубокой вытяжки с сохранением мелкозернистой структуры в месте изгиба и высоким процентом выхода годного. В результате проведенных исследований показано, что при температурах работающей сетки 800, 1000, $1100^{\circ} \mathrm{C}$ у сплава ВЦУН10-1 предел прочности в 2,5 - 4 раза выше, чем у сплава ЦН-25 и гафния. ВЦУН10-1 обеспечивает повышенную не менее чем в два раза долговечность электронных пушек при использовании в качестве электродов катодно-сеточного узла по сравнению с узлами с сетками из гафния.
\end{abstract}

Ключевые слова: антиэмиссионный материал, фазовый состав, прочность, формоустойчивость. 


\section{1. Введение}

Электроды катодно-сеточных узлов электровакуумных приборов изготавливают с применением антиэмиссионных материалов, способных подавлять эмиссию продуктов испарения катода с низкой работой выхода электронов [1].

Механизм подавления эмиссии имеет разную природу. Так, золото и платина с продуктами испарения катода образуют интерметаллидные соединения. Покрытия из золота применяют для катодов с невысокой рабочей температурой и с рабочей температурой сетки не более $500^{\circ} \mathrm{C}[1,2]$.

Механизм подавления эмиссии циркония, титана, гафния связан с разложением слоя оксида бария $\mathrm{BaO}$. Оксидные компоненты диффундируют в покрытие, при этом барий, материал с низкой работой выхода, быстро испаряется с поверхности электрода из антиэмиссионного материала. Вторичная эмиссия с сеток при этом существенно уменьшается [1-3].

В катодно-сеточных узлах, рабочая температура катодов которых равна $1000-1100^{\circ} \mathrm{C}$, применяют сетки из молибдена с покрытием из циркония или титана или сетки без покрытия из гафния или сплава ЦН-25.

Однако температуры рекристаллизации материалов сеток из молибдена $\left(900-1000^{\circ} \mathrm{C}\right)$, гафния $\left(950-1100^{\circ} \mathrm{C}\right)$ и циркония $\left(750^{\circ} \mathrm{C}\right)$ близки к температуре работающей сетки. В связи с этим, электровакуумные приборы выходят из строя в результате потери формоустойчивости сеток в процессе работы. При этом происходит их локальный перегрев, вплоть до полного выгорания. Поэтому применение в катодно-сеточных узлах антиэмиссионного материала, обладающего более высокой температурой рекристаллизации и более высокой формоустойчивостью, является актуальной и значимой проблемой.

Работа по изготовлению сплава выполнялась совместно с предприятием ГИРЕДМЕТ (г. Москва) по техническому заданию авторов, соответствующему требованиям к электродам катодно-сеточных узлов электровакуумных приборов.

В результате разработан сплав на основе ванадия, легированный ниобием (10\%), цирконием ( 1\%) и углеродом ( 1\%) - ВЦУН10-1. Ванадий близок по свойствам к материалам, обладающим высокими антиэмиссионными свойствами, таким как гафний, титан или цирконий. Легирующие добавки, ниобий и цирконий, согласно [4], также обладают высокими антиэмиссионными свойствами. Кроме того, они обеспечивают упрочнение сплава в результате образования карбидов и эффективно снижают пластическую деформацию материала путем торможения передвижения дислокаций.

Настоящая статья посвящена исследованию свойств ВЦУН10-1, используемого в качестве заготовок сеток и электродов катодно-сеточных узлов. Приведены сравнительные исследования свойств сплава со свойствами широко используемого гафния и сплава на основе циркония ЦН-25.

\section{2. Методика исследований}

Рентгенофазовый анализ выполняли на установке ДРОН-4 в $\mathrm{CuK} \alpha$-излучении.

Испытания на растяжение проводили на установке ИР5082-100, высокотемпературные испытания на растяжение в вакууме - на испытательной машине ПВ-312М, испытания на глубину сферической лунки - на установке ПТЛ. Отжиг производили в вакуумной электропечи сопротивления СНВЭ-16/13. Для улучшения вакуума $6,65 \cdot 10^{-3}$ Па создавали микрозону путем плотной укладки заготовок, защиты молибденовыми прокладками с двух сторон и упаковки полученной стопки в обезгаженную танталовую фольгу толщиной 0,02 мм. Фольга обеспечивала локальное улучшение вакуума. Газосодержание (массовая доля, \%) в исходных (деформированных) и отожженных листах толщиной 0,3 мм анализировали методом вакуумплавления по ГОСТ 22720.1-77 на специально подготовленных образцах.

\section{3. Результаты исследований и их обсуждение}

Детали и заготовки для сеток катодно-сеточных узлов изготавливают при комнатной температуре из отожженных в вакууме $6,65 \cdot 10^{-3}-1,33 \cdot 10^{-4}$ Па заготовок, в то время как рабочая температура изделий из этих материалов составляет около $900^{\circ} \mathrm{C}$ в более высоком вакууме (не ниже $4 \cdot 10^{-5}$ Па). В связи с этим большое значение имеют структура, прочностные и пластические характеристики заготовок материалов в условиях комнатных температур и прочность готовых изделий при повышенных температурах.

Рентгеноструктурный анализ образцов ВЦУН10-1 в исходном состоянии и после испытаний в качестве сеток показал, что сплав относится к высокопрочным, гетерофазным сплавам с упрочнением карбидами ванадия $\mathrm{VC}$ и циркония $\mathrm{ZrC}(\mathrm{O})$. Введение ниобия с целью упрочнения матрицы приводит к его растворению в решетке ванадия с образованием твердого раствора, что также способствует повышению его прочности.

Повышение растворения кислорода остаточных газов печей в гафнии, сплавах ЦН-25 и ВЦУН10-1, при отжиге заготовок в вакууме $6,65 \cdot 10^{-3}$ Па, показано методом вакуумплавления (табл. 1).

Табл. 1. Содержание кислорода (масс. \%) в деформированных и отожженных в вакууме $6,65 \cdot 10^{-3}$ Па листовых заготовках толщиной $0,3 \cdot 10^{-3} \mathrm{M}$.

Table 1. Amount of oxygen (mass. \%) for the sheet samples up to $0,3 \cdot 10^{-3} \mathrm{~m}$ thick, deformed and annealed in the vacuum $6,65 \cdot 10^{-3} \mathrm{~Pa}$.

\begin{tabular}{|c|c|c|}
\hline \multirow{2}{*}{$\begin{array}{c}\text { Материал } \\
\text { Material }\end{array}$} & \multicolumn{2}{|c|}{$\begin{array}{c}\text { Содержание кислорода (масс. \%) } \\
\text { Amount of oxygen (mass. \%) }\end{array}$} \\
\cline { 2 - 3 } & $\begin{array}{c}\text { деформированные } \\
\text { deformed }\end{array}$ & $\begin{array}{c}\text { отожженные } \\
\text { annealed }\end{array}$ \\
\hline $\begin{array}{c}\text { Сплав ЦН-25 } \\
\text { ZrNb-25 alloy }\end{array}$ & 0,02 & 0,06 \\
\hline $\begin{array}{c}\text { Гафний ГФИ } \\
\text { Наfnium GFI }\end{array}$ & 0,03 & 0,06 \\
\hline $\begin{array}{c}\text { Сплав ВЦУН10-1 } \\
\text { VZrCNb10-1 alloy }\end{array}$ & 0,02 & 0,07 \\
\hline
\end{tabular}


Из табл. 1 видно, что разработанный сплав ВЦУН10-1 относится к материалам, активно растворяющим в себе кислород остаточных газов печей при повышенных температурах в условиях вакуума. Таким образом, сплав ВЦУН10-1, так же как гафний и цирконий, обладает антиэмиссионными свойствами, что позволяет применять его в качестве сеток катодно-сеточных узлов.

Гафний и сплав ЦН-25, как видно из табл. 2, при растворении остаточных газов печей, изменяют свой фазовый состав. В результате рентгеноструктурного анализа установлено, что в образцах из гафния и сплава ЦН-25 после отжига, в используемом в производстве для отжига заготовок вакууме $6,65 \cdot 10^{-3}$ Па, образуется карбид и оксид соответствующего металла. При этом в результате визуального анализа обнаружено наличие на поверхности гафния плотной пленки темно-серого цвета и незначительное окисление образцов из сплава ЦН-25. В аналогичных образцах из сплава ВЦУН10-1 наличие окислов визуально и рентгеноструктурным методом не было обнаружено (табл. 2). Структура образцов из сплава ВЦУН10-1 при отжиге в исследуемом вакууме не изменяется.

Сравнение данных, приведенных в табл. 3, показывает, что гафний наиболее интенсивно изменяет свои свойства при растворении остаточных газов печи. Более низкий вакуум при отжиге приводит к уменьшению глу- бины вытяжки, значительному снижению пластичности и повышению прочности материала.

Результаты таблиц 1, 2 и 3 показывают, что сплав ВЦУН10-1 способен растворить большее количество кислорода без изменения фазового состава и значительного изменения свойств, чем гафний и сплав ЦН-25. Эта способность свидетельствует о том, что заготовки деталей и сборочные единицы из гафния и сплава циркония требуют более тщательной защиты от остаточных газов вакуумных печей, чем из сплава ВЦУН10-1 в процессе выполнения отжига.

Даже при применении защиты поверхности заготовок из гафния и сплава ЦН-25 при отжиге, заготовки из сплава ВЦУН10-1 обладают более высокой прочностью при хороших показателях пластичности. Это позволяет изготавливать детали с малыми радиусами кривизны $\left(R_{0,1 \max }\right)$ методами глубокой вытяжки с сохранением мелкозернистой структуры листа после отжига в месте его изгиба и высоким процентом выхода годного. Этого не удается достичь при изготовлении сеток из мелкозернистого гафния даже с радиусом кривизны $R_{0,6 \max }$.

Прочность ВЦУН10-1 при комнатной температуре в 1,5 раза выше, чем у гафния, при практически одинаковой пластичности.

Наличие мелкодисперсных карбидов в сплаве ВЦУН10-1 определяет высокую жаропрочность сплава.

Табл. 2. Рентгеноструктурный анализ влияния вакуума при отжиге заготовок на фазовый состав гафния и сплавов ЦН-25 и ВЦУН10-1.

Table 2. The X-ray diffraction analysis for the influence of vacuum on the phase composition of hafnium, and ZrNb-25 and VZrCNb10-1 alloys under annealing.

\begin{tabular}{|c|c|c|c|c|}
\hline $\begin{array}{l}\text { Марка } \\
\text { материала } \\
\text { Material } \\
\text { brand }\end{array}$ & $\begin{array}{c}\text { Толщина образцов, } \\
h \cdot 10^{3}, \mathrm{M} \\
\text { Thickness of samples, } \\
h \cdot 10^{3}, \mathrm{~m}\end{array}$ & $\begin{array}{c}\text { Режим } \\
\text { отжига } \\
\text { Annealing } \\
\text { mode }\end{array}$ & $\begin{array}{c}\text { Фазовый состав после отжига } \\
\text { в вакууме } 6,65 \cdot 10^{-3} \text { Па } \\
\text { Phase structure after annealing } \\
\text { in vacuum } 6,65 \cdot 10^{-3} \mathrm{~Pa}\end{array}$ & $\begin{array}{c}\text { Фазовый состав после отжига } \\
\text { в вакууме } 1,33 \cdot 10^{-4} \text { Па } \\
\text { Phase structure after annealing } \\
\text { in vacuum } 1,33 \cdot 10^{-4} \mathrm{~Pa}\end{array}$ \\
\hline $\begin{array}{l}\text { ГФИ-1 } \\
\text { GFI }\end{array}$ & 0,3 & $\begin{array}{l}950^{\circ} \mathrm{C} \\
30 \mathrm{~min}\end{array}$ & $\mathrm{Hf}, \mathrm{HfO}_{2}, \mathrm{HfC}$ & Hf, HfC \\
\hline $\begin{array}{c}\text { ЦH-25 } \\
\mathrm{ZrNb}-25\end{array}$ & 0,3 & $\begin{array}{l}750^{\circ} \mathrm{C} \\
30 \mathrm{~min}\end{array}$ & $\begin{array}{c}\mathrm{Zr} \text {, твердый раствор на основе } \\
\mathrm{Nb}, \mathrm{ZrO}_{2}, \mathrm{ZrC} \\
\mathrm{Zr} \text {, solid solution on the basis of } \\
\mathrm{Nb}, \mathrm{ZrO}_{2}, \mathrm{ZrC}\end{array}$ & $\begin{array}{c}\mathrm{Zr} \text {, твердый раствор на основе } \\
\mathrm{Nb}, \mathrm{ZrC} \\
\mathrm{Zr} \text {, solid solution on the basis of } \\
\mathrm{Nb}, \mathrm{ZrC}\end{array}$ \\
\hline \multirow{2}{*}{$\begin{array}{l}\text { ВЦУН10-1 } \\
\text { VZrCNb10-1 }\end{array}$} & 0,3 & \multirow{2}{*}{$\begin{array}{l}1250^{\circ} \mathrm{C} \\
30 \mathrm{~min}\end{array}$} & \multirow{2}{*}{\multicolumn{2}{|c|}{$\begin{array}{l}\text { твердый раствор на основе } \mathrm{V}, \mathrm{VC}, \mathrm{ZrC}(\mathrm{O}) \\
\text { solid solution on the basis of } \mathrm{V}, \mathrm{VC}, \mathrm{ZrC}(\mathrm{O})\end{array}$}} \\
\hline & 0,1 & & & \\
\hline
\end{tabular}

Табл. 3. Влияние вакуума в процессе отжига на предел прочности $\left(\sigma_{\mathrm{B}}\right)$, относительное удлинение $(\delta)$ и глубину вытяжки $(h)$ образцов из гафния и сплавов ЦН-25 и ВЦУН10-1.

Table 3. Influence of vacuum during annealing on the stress limit $\left(\sigma_{\mathrm{B}}\right)$, tensile elongation $(\delta)$, and deep-drawing ration $(h)$ of hafnium and the ZrNb-25 and VZrCNb10-1 alloys.

\begin{tabular}{|c|c|c|c|c|c|c|c|c|}
\hline \multirow{2}{*}{$\begin{array}{c}\text { Марка } \\
\text { материала } \\
\text { Material } \\
\text { identification }\end{array}$} & \multirow{2}{*}{$\begin{array}{c}\text { Толщина образцов, } \\
h \cdot 10^{3}, \mathrm{~m} \\
\text { Sample thickness, } \\
h \cdot 10^{3}, \mathrm{~m}\end{array}$} & \multirow{2}{*}{$\begin{array}{c}\text { Режим } \\
\text { термообработки } \\
\text { Heat treatment } \\
\text { mode }\end{array}$} & \multicolumn{3}{|c|}{$\begin{array}{l}6,65 \cdot 10^{-3} \Pi \mathrm{Ma} \\
6,65 \cdot 10^{-3} \mathrm{~Pa}\end{array}$} & \multicolumn{3}{|c|}{$\begin{array}{l}1,33 \cdot 10^{-4} \prod \mathrm{a} \\
1,33 \cdot 10^{-4} \mathrm{~Pa}\end{array}$} \\
\hline & & & $\begin{array}{l}\sigma_{\mathrm{B}} \cdot 10^{-1}, \mathrm{MПа} \\
\sigma_{\mathrm{B}} \cdot 10^{-1}, \mathrm{MPa}\end{array}$ & $\delta, \%$ & $\begin{array}{l}h \cdot 10^{3} \mathrm{M} \\
h \cdot 10^{3} \mathrm{~m}\end{array}$ & $\begin{array}{c}\sigma_{\mathrm{B}} \cdot 10^{-1}, \mathrm{MПа} \\
\sigma_{\mathrm{B}} \cdot 10^{-1}, \mathrm{MPa}\end{array}$ & $\delta, \%$ & $\begin{array}{l}h \cdot 10^{3} \mathrm{M} \\
h \cdot 10^{3} \mathrm{~m}\end{array}$ \\
\hline \multirow{2}{*}{$\begin{array}{c}\text { ГФИ-1 } \\
\text { GFI }\end{array}$} & 0,3 & \multirow{2}{*}{$\begin{array}{l}950^{\circ} \mathrm{C}, \\
30 \mathrm{~min} \\
\end{array}$} & 56,8 & 4,8 & 1,06 & 51,1 & 15,0 & 2,07 \\
\hline & 0,1 & & 47,0 & 7,0 & 1,2 & 40,5 & 12,5 & 1,7 \\
\hline \multirow{2}{*}{$\begin{array}{c}\text { ЦН-25 } \\
\mathrm{ZrNb}-25\end{array}$} & 0,3 & \multirow{2}{*}{$\begin{array}{l}750^{\circ} \mathrm{C}, \\
30 \mathrm{~min}\end{array}$} & 66,5 & 8,0 & - & 62,1 & 9,2 & - \\
\hline & 0,1 & & 68,5 & 5,0 & 2,1 & 65,0 & 5,5 & 2,6 \\
\hline \multirow{2}{*}{$\begin{array}{c}\text { ВЦУН10-1 } \\
\text { VZrCNb10-1 }\end{array}$} & 0,3 & \multirow{2}{*}{$\begin{array}{l}1250^{\circ} \mathrm{C} \\
30 \mathrm{~min}\end{array}$} & 63,3 & 15,0 & 3,06 & 62,2 & 15,2 & 3,12 \\
\hline & 0,1 & & 62,2 & 9,5 & 2,64 & 61,5 & 15,0 & 2,85 \\
\hline
\end{tabular}


Его температура первичной рекристаллизации составляет $1250^{\circ} \mathrm{C}$, а следовательно, температура отжига значительно выше, чем у гафния и сплава ЦН-25 (табл. 2, 3).

При повышенных температурах, в интервале $800-1100^{\circ} \mathrm{C}$ и вакууме $1,33 \cdot 10^{-4}$ Па, прочностные характеристики сплава ВЦУН10-1 в 2,5-4 раза выше, чем у гафния и сплава ЦН-25 (табл. 4), что должно способствовать повышению формоустойчивости материала при долговечной работе в качестве электродов катодносеточных узлов.

Испытания, выполненные в макетах катодно-сеточных узлов с технологическим анодом, показали (рис. 1), что долговечность узлов при использовании в качестве сеток сплава ВЦУН10-1 повышается не менее чем в 2 раза по сравнению с узлами с сетками из гафния. Критерием долговечности является ток утечки $i_{\text {ут }}$ между катодом и управляющей сеткой, который не должен превышать 0,1 мА.
Табл. 4. Механические свойства листовых образцов сплавов ВЦУН10-1, ЦН-25 и гафния ГФИ-1 толщиной 0,3 мм при комнатной температуре $\left(20^{\circ} \mathrm{C}\right)$ и температуре работающей сетки $\left(800-1100^{\circ} \mathrm{C}\right)$

Table 4. Mechanical properties of the sheet sample alloys VZrCNb10-1, ZrNb-25 and the hafnium GFI-1, 0,3 mm thick, at the room temperature $\left(20^{\circ} \mathrm{C}\right)$ and the working grid temperature $\left(800-1100^{\circ} \mathrm{C}\right)$

\begin{tabular}{|c|c|c|c|c|c|}
\hline \multirow{3}{*}{$\begin{array}{l}\text { Материал } \\
\text { Material }\end{array}$} & \multirow{3}{*}{$\begin{array}{c}\text { Режим } \\
\text { термообработки } \\
\text { Heat treatment } \\
\text { mode }\end{array}$} & \multicolumn{4}{|c|}{$\sigma_{\mathrm{B}} \cdot 10^{-1}, \mathrm{MPa}$} \\
\hline & & \multicolumn{4}{|c|}{$\begin{array}{c}\text { Температура испытаний, }{ }^{\circ} \mathrm{C} \\
\text { Temperature of tests, }{ }^{\circ} \mathrm{C}\end{array}$} \\
\hline & & 20 & 800 & 1000 & 1100 \\
\hline $\begin{array}{c}\text { ГФИ-1 } \\
\text { GFI }\end{array}$ & $\begin{array}{l}950^{\circ} \mathrm{C} \\
30 \mathrm{~min}\end{array}$ & 45,9 & 6,5 & 5,5 & 3,2 \\
\hline $\begin{array}{l}\text { ЦH-25 } \\
\mathrm{ZrNb}-25\end{array}$ & $\begin{array}{l}750^{\circ} \mathrm{C} \\
30 \mathrm{~min}\end{array}$ & 62,0 & - & 8,3 & - \\
\hline $\begin{array}{c}\text { ВЦУН10-1 } \\
\text { VZrCNb10-1 }\end{array}$ & $\begin{array}{l}1250^{\circ} \mathrm{C} \\
30 \mathrm{~min}\end{array}$ & 68,0 & - & 20,6 & 11,5 \\
\hline
\end{tabular}

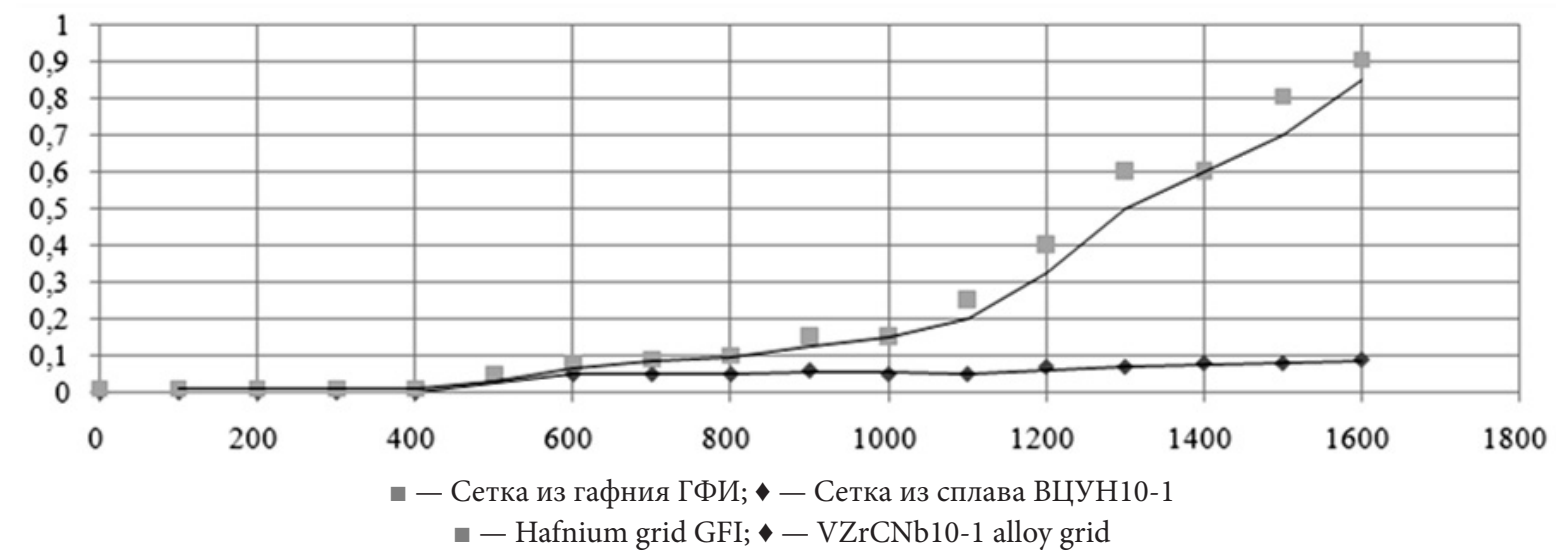

Pис. 1. Изменение тока утечки макетов электронной пушки с сетками из разных антиэмиссионных материалов при испытаниях на долговечность при температуре катода $1070^{\circ} \mathrm{C}$. Толщина теневой сетки $-0,1$ мм, управляющей $-0,15$ мм.

Fig. 1. Changes of the leakage current in electronic gun models with grids of various anti-emission materials during endurance tests with the cathode temperature at $1070^{\circ} \mathrm{C}$. Thickness of the shadow grid $-0,1 \mathrm{~mm}$, thickness of the control grid $-0,15 \mathrm{~mm}$.

\section{4. Заключение}

Показано, что при комнатной температуре предел прочности сплавов ВЦУН10-1 и ЦН-25 в 1,5 раза выше, чем у гафния. А при температурах, соответствующих температурам работающей сетки, у сплава ВЦУН10-1 он в 2,5 - 4 раза выше, чем у сплава ЦН-25 и гафния.

Разработанный сплав на основе ванадия ВЦУН10-1 обладает лучшей способностью сохранять прочностные и технологические характеристики при отжиге в вакууме $6,65 \cdot 10^{-3}$ Па, чем гафний и сплав ЦН-25, при этом не требует специальной защиты поверхности заготовок при отжиге.

Показано, что применение сплава ВЦУН10-1, вместо гафния, приводит к значительному, не менее чем в 2 раза, повышению долговечности электронной пушки.

Благодарность/Aknowledgements. Часть исследований выполнена при поддержке Гранта Президента РФ МД-1403.2017.8.

\section{Литература/References}

1. B.Ch. Dubua, O.K. Cultashev, O.V. Polivnikova. Electronic Engineering. Series 1, Microwave Engineering. 4(497), 3-22 (2008). (in Russian) [Б. Ч. Дюбуа, О. К. Култашев, О. В. Поливникова. Электронная техника. Сер. 1, СВЧ-техника. 4(497), 3 - 22 (2008).]

2. B.Ch. Dubua. Radio electronics questions. Series 1: Electronics. 12. 61 (1960). (in Russian) [Б.Ч. Дюбуа. Вопросы радиоэлектроники. Сер.1. Электроника. 12. $61(1960)$.]

3. A.M. Sokolov, A.N. Kargin, O. A. Morozov. Electronic Engineering. Series 1, Microwave Engineering. 1(508). 64-69 (2011). (in Russian) [A. М. Соколов, А.Н. Каргин, О.А. Морозов. Электронная техника. Сер. 1, СВЧ-техника. 1(508). 64 - 69 (2011).]

4. Zh. N. Babanov, V.I. Kozlov, A.A. Andreev. Electronic equipment. Series 6. Materials. 8(320). 14 - 17 (1980). (in Russian) [Ж.Н. Бабанов, В.И. Козлов, А. А. Андреев. Электронная техника. Сер.6. Материалы. 8(320). 14 - 17 (1980) .] 